\title{
Cusps of Bishop Spherical Indicatrixes and Their Visualizations
}

\author{
Haiming Liu ${ }^{1,2}$ and Donghe Pei ${ }^{1}$ \\ ${ }^{1}$ School of Mathematics and Statistics, Northeast Normal University, Changchun 130024, China \\ ${ }^{2}$ School of Science, Mudanjiang Normal University, Mudanjiang 157011, China
}

Correspondence should be addressed to Donghe Pei; peidh340@nenu.edu.cn

Received 15 October 2013; Accepted 9 December 2013

Academic Editor: Bo Shen

Copyright (C) 2013 H. Liu and D. Pei. This is an open access article distributed under the Creative Commons Attribution License, which permits unrestricted use, distribution, and reproduction in any medium, provided the original work is properly cited.

\begin{abstract}
The main result of this paper is using Bishop Frame and "Type-2 Bishop Frame" to study the cusps of Bishop spherical images and type-2 Bishop spherical images which are deeply related to a space curve and to make them visualized by computer. We find that the singular points of the Bishop spherical images and type-2 Bishop spherical images correspond to the point where Bishop curvatures and type-2 Bishop curvatures vanished and their derivatives are not equal to zero. As applications and illustration of the main results, two examples are given.
\end{abstract}

\section{Introduction}

This paper is written as one of the research projects on visualization of singularities of submanifolds generated by regular curves embedded in Euclidean 3-space by using Bishop Frame. It is well known that Bishop introduced the Relatively Parallel Adapted Frame of regular curves embedded in Euclidean 3-space and this frame was widely applied in the area of Biology and Computer Graphics; see [1-5]. For instance, in [6], we introduced some detailed applications of Bishop Frame, so we omit them here. Inspired by the work of Bishop, in [7], the authors introduced a new version of Bishop Frame using a common vector field as binormal vector field of a regular curve and called this frame "Type-2 Bishop Frame." They also introduced two new spherical images and called them type-2 Bishop spherical images. We know that the properties of geometric objects are independent of the choice of the coordinate systems. But the researchers $[1,7-13]$ found that, when they adopted these frames, there would be some new geometric objects such as Bishop spherical images, Bishop Daroux image, and type-2 Bishop spherical images. Although Bishop spherical images and type-2 Bishop spherical images have been well studied from the standpoint of differential geometry when they are regular spherical curves, there are little papers on their singularities. Actually, sometimes they are singular, for example, $[7,10]$. Two questions are what about their singularities and how to recognize the types of the singularities? Thus the current study hopes to answer these questions and it is inspired by the works of Bishop [1], Yllmaz et al. [7], Pei and Sano [14] and Wang et al. [15]. On the other hand, for the reason that the vector parameterized equations of Bishop spherical images and type-2 Bishop spherical images are very complicated (see $[7,10])$, it is very hard to recognize their singular points by normal way. In this paper, we will give a simple sufficient condition to describe their singular points by using the technique of singularity theory. To do this, we hope that Bishop spherical images and type-2 Bishop spherical images can be seen as the discriminants of unfolding of some functions. In this paper, adopting Bishop Frame [1] and "Type-2 Bishop Frame" [7] as the basic tools, we construct Bishop normal indicatrix height functions (denoted by $H_{i}$ : $I \times S^{2} \rightarrow \mathbb{R}, H_{i}(s, \mathbf{v})=\left\langle\mathbf{N}_{i}(s), \mathbf{v}\right\rangle$, where $i=1,2$ and $\mathbf{N}_{i}(s)$ is the first Bishop spherical indicatrix or the second Bishop spherical indicatrix and $H_{j}: I \times S^{2} \rightarrow \mathbb{R}$, $H_{j}(s, \mathbf{v})=\left\langle\zeta_{j}(s), \mathbf{v}\right\rangle$, where $j=3,4$ and $\zeta_{j}(s)$ is the first type2 Bishop spherical indicatrix or the second type-2 Bishop spherical indicatrix) locally around the point $\left(s_{0}, \mathbf{v}_{0}\right)$. These functions are the unfolding of these singularities in the local neighbourhood of $\left(s_{0}, \mathbf{v}_{0}\right)$ and depend only on the germ that they are unfolding. We create these functions by varying a fixed point $\mathbf{v}$ in these Bishop normal indicatrix height 
functions to get some families of functions. We show that these singularities are versally unfolded by the families of Bishop normal indicatrix height functions. If the singularity of $h_{i v}=H_{i}(s, \mathbf{v})$ and $h_{j v}=H_{j}(s, \mathbf{v})$ is $A_{k}$-type $(k=$ $1,2)$ and the corresponding 2-parameter unfolding is versal, then applying the theory of singularity [14, 15], we know that discriminant set of the 2-parameter unfolding is locally diffeomorphic to a line or a cusp; thus, we finished the classification of singularities of the Bishop spherical images and type-2 Bishop spherical images, because the discriminant sets of the unfolding are precisely the Bishop spherical images and type-2 Bishop spherical images. Moreover, we see that the $A_{k}$-singularity $(k=1,2)$ of $h_{i v}$ and $h_{j v}$ is closely related to Bishop curvatures and type-2 Bishop curvatures. The singular points of the Bishop spherical images and type2 Bishop spherical images correspond to the point where Bishop curvatures and type-2 Bishop curvatures vanished and their derivatives are not equal to zero. Thus, we get the main results in this paper which are stated in Theorem 1 . It is important that the properties of those functions related with space curve $\gamma(s)$ needed to be generic [15]. Once we proved that the properties of those functions were generic, we could deduce that all singularities were stable under small perturbations for our family of functions. By considering transversality, we prove that these properties given by us are generic. As applications of our main results, we give two examples.

The rest of this paper is organized as follows. Firstly, we introduce some basic concepts and the main results in the next two sections. Then, we introduce four different families of functions on $\gamma$ that will be useful to the study of Bishop spherical images and type-2 Bishop spherical images. Afterwards, some general results on the singularity theory are used for families of function germs and the main results are proved. Finally, we give two examples to illustrate the main results and the conclusion of the work is drawn.

\section{Preliminaries and Notations}

In this section, we will introduce the notions of Bishop Frame, “Type-2 Bishop Frame," Bishop spherical images, and type-2 Bishop spherical images of unit speed regular curve. Let $\gamma=$ $\gamma(s)$ be a regular unit speed Frenet curve in $\mathbb{E}^{3}$. We know that there exist accompanying three frames called Frenet frame for Frenet curve. Denote by $(\mathbf{T}(s), \mathbf{N}(s), \mathbf{B}(s))$ the moving Frenet frame along the unit speed Frenet curve $\gamma(s)$. Then, the Frenet formulas are given by

$$
\left(\begin{array}{l}
\mathbf{T}^{\prime}(s) \\
\mathbf{N}^{\prime}(s) \\
\mathbf{B}^{\prime}(s)
\end{array}\right)=\left(\begin{array}{ccc}
0 & k(s) & 0 \\
-k(s) & 0 & \tau(s) \\
0 & -\tau(s) & 0
\end{array}\right)\left(\begin{array}{l}
\mathbf{T}(s) \\
\mathbf{N}(s) \\
\mathbf{B}(s)
\end{array}\right) .
$$

Here, $k(s)$ and $\tau(s)$ are called curvature and torsion, respectively [16]. The Bishop Frame of the $\gamma(s)$ is expressed by the alternative frame equations:

$$
\left(\begin{array}{c}
\mathbf{T}^{\prime}(s) \\
\mathbf{N}_{1}^{\prime}(s) \\
\mathbf{N}_{2}^{\prime}(s)
\end{array}\right)=\left(\begin{array}{ccc}
0 & k_{1}(s) & k_{2}(s) \\
-k_{1}(s) & 0 & 0 \\
-k_{2}(s) & 0 & 0
\end{array}\right)\left(\begin{array}{c}
\mathbf{T}(s) \\
\mathbf{N}_{1}(s) \\
\mathbf{N}_{2}(s)
\end{array}\right) \text {. }
$$

Here, we will call the set $\left(\mathbf{T}(s), \mathbf{N}_{1}(s), \mathbf{N}_{2}(s)\right)$ as Bishop Frame and $k_{1}(s)=\left\langle\mathbf{T}^{\prime}(s), \mathbf{N}_{1}(s)\right\rangle$ and $k_{2}(s)=\left\langle\mathbf{T}^{\prime}(s), \mathbf{N}_{2}(s)\right\rangle$ as Bishop curvatures. The relation matrix can be expressed as

$$
\left(\begin{array}{l}
\mathbf{T}(s) \\
\mathbf{N}(s) \\
\mathbf{B}(s)
\end{array}\right)=\left(\begin{array}{ccc}
1 & 0 & 0 \\
0 & \cos \theta(s) & \sin \theta(s) \\
0 & -\sin \theta(s) & \cos \theta(s)
\end{array}\right)\left(\begin{array}{c}
\mathbf{T}(s) \\
\mathbf{N}_{1}(s) \\
\mathbf{N}_{2}(s)
\end{array}\right) .
$$

One can show that

$$
\begin{gathered}
k(s)=\sqrt{k_{1}^{2}(s)+k_{2}^{2}(s)}, \\
\theta(s)=\arctan \left(\frac{k_{2}(s)}{k_{1}(s)}\right), \quad \text { where } k_{1}(s) \neq 0, \tau(s)=\frac{d \theta(s)}{d s},
\end{gathered}
$$

so that $k_{1}(s)$ and $k_{2}(s)$ effectively correspond to a Cartesian coordinate system for the polar coordinates $k(s)$ and $\theta(s)$ with $\theta=\int \tau(s) d s$. Here, Bishop curvatures are also defined by

$$
\begin{aligned}
& k_{1}(s)=k(s) \cos \theta(s), \\
& k_{2}(s)=k(s) \sin \theta(s) .
\end{aligned}
$$

The orientation of the parallel transport frame includes the arbitrary choice of integration constant $\theta_{0}$, which disappears from $\tau$ (and hence from the Frenet frame) due to the differentiation [1].

The "Type-2 Bishop Frame" of the $\gamma(s)$ is defined by the alternative frame equations; see [7],

$$
\left(\begin{array}{l}
\boldsymbol{\zeta}_{1}^{\prime}(s) \\
\zeta_{2}^{\prime}(s) \\
\mathbf{B}^{\prime}(s)
\end{array}\right)=\left(\begin{array}{ccc}
0 & 0 & -\epsilon_{1}(s) \\
0 & 0 & -\epsilon_{2}(s) \\
\epsilon_{1}(s) & \epsilon_{2}(s) & 0
\end{array}\right)\left(\begin{array}{l}
\zeta_{1}(s) \\
\zeta_{2}(s) \\
\mathbf{B}(s)
\end{array}\right) .
$$

The relation matrix between Frenet-Serret and "Type-2 Bishop Frame" can be expressed as

$$
\left(\begin{array}{l}
\mathbf{T}(s) \\
\mathbf{N}(s) \\
\mathbf{B}(s)
\end{array}\right)=\left(\begin{array}{ccc}
\sin \theta(s) & -\cos \theta(s) & 0 \\
\cos \theta(s) & \sin \theta(s) & 0 \\
0 & 0 & 1
\end{array}\right)\left(\begin{array}{l}
\zeta_{1}(s) \\
\zeta_{2}(s) \\
\mathbf{B}(s)
\end{array}\right) .
$$

Here, the type-2 Bishop curvatures are defined by

$$
\begin{aligned}
& \epsilon_{1}(s)=-\tau(s) \cos \theta(s), \\
& \epsilon_{2}(s)=-\tau(s) \sin \theta(s) .
\end{aligned}
$$

We will call the set $\left(\boldsymbol{\zeta}_{1}(s), \boldsymbol{\zeta}_{2}(s), \mathbf{B}(s)\right)$ "Type-2 Bishop Frame" which is properly oriented and $\epsilon_{1}(s)=\left\langle\mathbf{B}^{\prime}(s), \zeta_{1}(s)\right\rangle$ and $\epsilon_{2}(s)=\left\langle\mathbf{B}^{\prime}(s), \zeta_{2}(s)\right\rangle$ type-2 Bishop curvatures. One also can show that

$$
\kappa(s)=\frac{d \theta(s)}{d s},
$$

so that $\epsilon_{1}(s)$ and $\epsilon_{2}(s)$ also effectively correspond to a Cartesian coordinate system for the polar coordinates $\tau(s)$, $\theta(s)$ with $\theta=\int \kappa(s) d s$.

The following notions are the main objects in this paper. The unit sphere with center in the origin in the space $\mathbb{E}^{3}$ is defined by

$$
S^{2}=\left\{\mathbf{x} \in \mathbb{E}^{3} \mid\langle\mathbf{x}, \mathbf{x}\rangle=1\right\} .
$$


Translating frame's vector fields to the center of unit sphere, we obtain Bishop spherical images or Bishop spherical indicatrixes. The first Bishop spherical indicatrix and the second Bishop spherical indicatrix are denoted by $\mathscr{F} \mathscr{B} \mathscr{N}(s)=\mathbf{N}_{1}(s)$ and $\mathcal{S} \mathscr{B} \mathscr{N}(s)=\mathbf{N}_{2}(s)$ separately. The first type-2 Bishop spherical indicatrix and the second type- 2 Bishop spherical indicatrix in [7] is denoted by $\mathscr{F} \mathscr{N} \mathscr{B} \mathscr{N}(s)=\zeta_{1}(s)$ and $\mathcal{S} \mathscr{N} \mathscr{B} \mathscr{N}(s)=\zeta_{2}(s)$ separately.

\section{Cusps of Bishop Spherical Indicatrixes and Their Visualizations}

The main results of this paper are in the following theorem.

Theorem 1. Let $\gamma: I \rightarrow \mathbb{E}^{3}$ be a regular unit speed curve. Then, one has the following.

(1) Suppose that $k_{2}(s) \neq 0$. Then, one have the following claims.

(a) The first Bishop spherical image $\mathbf{N}_{1}(s)$ is locally diffeomorphic to a line $\{0\} \times \mathbb{R}$ at $s_{0}$ if $k_{1}\left(s_{0}\right) \neq 0$.

(b) The first Bishop spherical image $\mathbf{N}_{1}(s)$ is locally diffeomorphic to the cusp $C$ at $s_{0}$ if $k_{1}\left(s_{0}\right)=0$ and $k_{1}^{\prime}\left(s_{0}\right) \neq 0$.

(2) Suppose that $k_{1}(s) \neq 0$. Then, one has the following claims.

(a) The second Bishop spherical image $\mathbf{N}_{2}(s)$ is locally diffeomorphic to a line $\{0\} \times \mathbb{R}$ at $s_{0}$ if $k_{2}\left(s_{0}\right) \neq 0$.

(b) The second Bishop spherical image $\mathbf{N}_{2}(s)$ is locally diffeomorphic to the cusp $C$ at $s_{0}$ if $k_{2}\left(s_{0}\right)=$ 0 and $k_{2}^{\prime}\left(s_{0}\right) \neq 0$.

(3) Suppose that $\epsilon_{2}(s) \neq 0$. Then, one has the following claims.

(a) The first type-2 Bishop spherical image $\zeta_{1}(s)$ is locally diffeomorphic to a line $\{0\} \times \mathbb{R}$ at $s_{0}$ if $\epsilon_{1}\left(s_{0}\right) \neq 0$.

(b) The first type-2 Bishop spherical image $\zeta_{1}(s)$ is locally diffeomorphic to the cusp $C$ at $s_{0}$ if $\epsilon_{1}\left(s_{0}\right)=$ 0 and $\epsilon_{1}^{\prime}\left(s_{0}\right) \neq 0$.

(4) Suppose that $\epsilon_{1}(s) \neq 0$. Then, one has the following claims.

(a) The second type-2 Bishop spherical image $\zeta_{2}(s)$ is locally diffeomorphic to a line $\{0\} \times \mathbb{R}$ at $s_{0}$ if $\epsilon_{2}\left(s_{0}\right) \neq 0$.

(b) The second type-2 Bishop spherical image $\zeta_{2}(s)$ is locally diffeomorphic to the cusp $C$ at $s_{0}$ if $\epsilon_{2}\left(s_{0}\right)=$ 0 and $\epsilon_{2}^{\prime}\left(s_{0}\right) \neq 0$, where the ordinary cusp is $C=$ $\left\{\left(x_{1}, x_{2}\right) \mid x_{1}^{2}=x_{2}^{3}\right\}$. The picture of cusp will be seen in Figure 1.

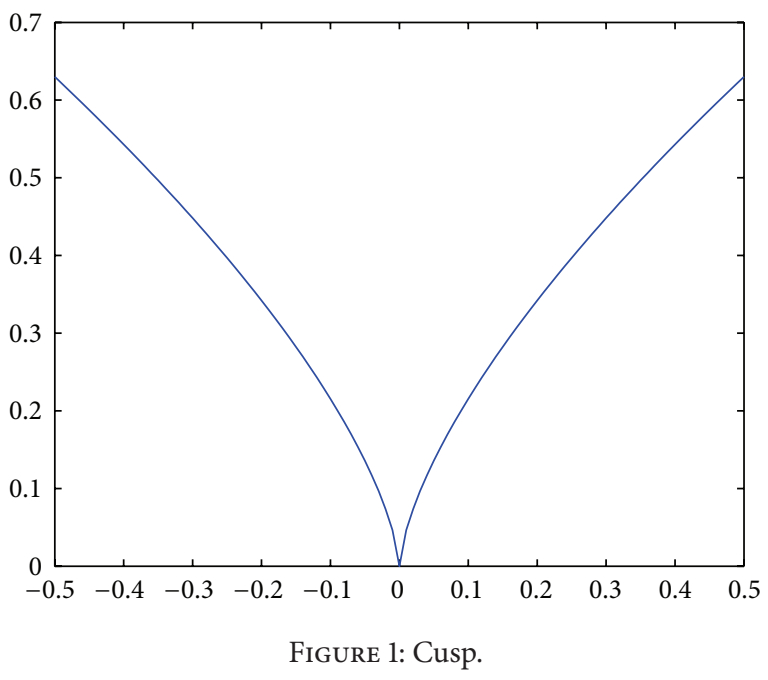

\section{Bishop Normal Indicatrix Height Functions}

In this section, we will introduce four different families of functions on $\gamma$ that will be useful to study the singular points of the Bishop spherical images and type-2 Bishop spherical images of unit speed regular curve. Let $\gamma: I \rightarrow \mathbb{E}^{3}$ be a regular unit speed curve. Now, we define two families of smooth functions on $I$ as follows:

$$
H_{i}: I \times S^{2} \longrightarrow \mathbb{R} \quad \text { by } H_{i}(s, \mathbf{v})=\left\langle\mathbf{N}_{i}(s), \mathbf{v}\right\rangle,
$$

where $i=1,2$. We call it the first (second) Bishop normal indicatrix height function for the case $i=1(i=2)$. For any $\mathbf{v} \in S^{2}$, we denote $h_{i v}(s)=H_{i}(s, \mathbf{v})$. We also define two families of smooth functions on $I$ as follows:

$$
H_{j}: I \times S^{2} \longrightarrow \mathbb{R} \text { by } H_{j}(s, \mathbf{v})=\left\langle\boldsymbol{\zeta}_{j}(s), \mathbf{v}\right\rangle,
$$

where $j=3,4$. We call it the first (second) type-2 Bishop normal indicatrix height function for the case $j=3(j=4)$. For any $\mathbf{v} \in S^{2}$, we denote $h_{j v}(s)=H_{j}(s, \mathbf{v})$. Then we have the following proposition.

Proposition 2. Let $\gamma: I \rightarrow \mathbb{E}^{3}$ be a regular unit speed curve. Then, one has the following.

(A) Suppose that $k_{1}(s) \neq 0$. Then, one has the following claims.

(1) $h_{1 v}(s)=0$ if and only if there are real numbers $\lambda$ and $\mu$ such that $\mathbf{v}=\lambda \mathbf{T}(s)+\mu \mathbf{N}_{2}(s)$ and $\lambda^{2}+\mu^{2}=$ 1.

(2) $h_{1 v}(s)=h_{1 v}^{\prime}(s)=0$ if and only if $\mathbf{v}=\mp \mathbf{N}_{2}(s)$.

(3) $h_{1 v}(s)=h_{1 v}^{\prime}(s)=h_{1 v}^{\prime \prime}(s)=0$ if and only if $\mathbf{v}=$ $\mp \mathbf{N}_{2}(s)$ and $k_{2}(s)=0$.

(4) $h_{1 v}(s)=h_{1 v}^{\prime}(s)=h_{1 v}^{\prime \prime}(s)=h_{1 v}^{(3)}(s)=0$ if and only if $\mathbf{v}=\mp \mathbf{N}_{2}(s)$ and $k_{2}(s)=k_{2}^{\prime}(s)=0$. 
(B) Suppose that $k_{2}(s) \neq 0$. Then, one has the following claims.

(1) $h_{2 v}(s)=0$ if and only if there are real numbers $\lambda$ and $\mu$ such that $\mathbf{v}=\lambda \mathbf{T}(s)+\mu \mathbf{N}_{1}(s)$ and $\lambda^{2}+\mu^{2}=$ 1.

(2) $h_{2 v}(s)=h_{2 v}^{\prime}(s)=0$ if and only if $\mathbf{v}=\mp \mathbf{N}_{1}(s)$.

(3) $h_{2 v}(s)=h_{2 v}^{\prime}(s)=h_{2 v}^{\prime \prime}(s)=0$ if and only if $\mathbf{v}=$ $\mp \mathbf{N}_{1}(s)$ and $k_{1}(s)=0$.

(4) $h_{2 v}(s)=h_{2 v}^{\prime}(s)=h_{2 v}^{\prime \prime}(s)=h_{2 v}^{(3)}(s)=0$ if and only if $\mathbf{v}=\mp \mathbf{N}_{1}(s)$ and $k_{1}(s)=k_{1}^{\prime}(s)=0$.

(C) Suppose that $\epsilon_{1}(s) \neq 0$. Then, one has the following claims.

(1) $h_{3 v}(s)=0$ if and only if there are real numbers $\lambda$ and $\mu$ such that $\mathbf{v}=\lambda \zeta_{2}(s)+\mu \mathbf{B}(s)$ and $\lambda^{2}+\mu^{2}=$ 1.

(2) $h_{3 v}(s)=h_{3 v}^{\prime}(s)=0$ if and only if $\mathbf{v}=\mp \zeta_{2}(s)$.

(3) $h_{3 v}(s)=h_{3 v}^{\prime}(s)=h_{3 v}^{\prime \prime}(s)=0$ if and only if $\mathbf{v}=$ $\mp \zeta_{2}(s)$ and $\epsilon_{2}(s)=0$.

(4) $h_{3 v}(s)=h_{3 v}^{\prime}(s)=h_{3 v}^{\prime \prime}(s)=h_{3 v}^{(3)}(s)=0$ if and only if $\mathbf{v}=\mp \zeta_{2}(s)$ and $\epsilon_{2}(s)=\epsilon_{2}^{\prime}(s)=0$.

(D) Suppose that $\epsilon_{2}(s) \neq 0$. Then, one has the following claims.

(1) $h_{4 v}(s)=0$ if and only if there are real numbers $\lambda$ and $\mu$ such that $\mathbf{v}=\lambda \zeta_{1}(s)+\mu \mathbf{B}(s)$ and $\lambda^{2}+\mu^{2}=$ 1 .

(2) $h_{4 v}(s)=h_{4 v}^{\prime}(s)=0$ if and only if $\mathbf{v}=\mp \zeta_{1}(s)$.

(3) $h_{4 v}(s)=h_{4 v}^{\prime}(s)=h_{4 v}^{\prime \prime}(s)=0$ if and only if $\mathbf{v}=$ $\mp \zeta_{1}(s)$ and $\epsilon_{1}(s)=0$.

(4) $h_{4 v}(s)=h_{4 v}^{\prime}(s)=h_{4 v}^{\prime \prime}(s)=h_{4 v}^{(3)}(s)=0$ if and only if $\mathbf{v}=\mp \zeta_{1}(s)$ and $\epsilon_{1}(s)=\epsilon_{1}^{\prime}(s)=0$.

Proof. (A) (1) If $h_{1 v}(s)=\left\langle\mathbf{N}_{1}(s), \mathbf{v}\right\rangle=0$, then we have that there are real numbers $\lambda$ and $\mu$ such that $\mathbf{v}=\lambda \mathbf{T}(s)+\mu \mathbf{N}_{2}(s)$. Moreover, in combination with $\mathbf{v} \in S^{2}$, which means $\lambda^{2}+\mu^{2}=$ 1 , it follows that $h_{1 v}(s)=0$ if and only if $\mathbf{v}=\lambda \mathbf{T}(s)+\mu \mathbf{N}_{2}(s)$ and $\lambda^{2}+\mu^{2}=1$.

(2) When $h_{1 v}(s)=0$, the assertion (2) follows from the fact that $h_{1 v}^{\prime}(s)=\left\langle k_{1}(s) \mathbf{T}(s), \mathbf{v}\right\rangle=k_{1}(s) \lambda$ and $k_{1}(s) \neq 0$. Thus, we get that $h_{1 v}(s)=h_{1 v}^{\prime}(s)=0$ if and only if $\mathbf{v}=\mp \mathbf{N}_{2}(s)$.

(3) When $h_{1 v}(s)=h_{1 v}^{\prime}(s)=0$, the assertion (3) follows from the fact that $h_{1 v}^{\prime \prime}(s)=\left\langle k_{1}^{\prime}(s) \mathbf{T}(s)+k_{1}^{2}(s) \mathbf{N}_{1}(s)+\right.$ $\left.k_{1}(s) k_{2}(s) \mathbf{N}_{2}(s), \mp \mathbf{N}_{2}(s)\right\rangle=\mp k_{1}(s) k_{2}(s)$ and $k_{1}(s) \neq 0$.

(4) Under the condition that $h_{1 v}(s)=h_{1 v}^{\prime}(s)=h_{1 v}^{\prime \prime}(s)=0$, this derivative is computed as follows: $h_{1 v}^{(3)}(s)=\left\langle\left(k_{1}^{\prime \prime}(s)+\right.\right.$ $\left.k_{1}^{3}(s)+k_{1}(s) k_{2}^{2}(s)\right) \mathbf{T}(s)+3 k_{1}(s) k_{1}^{\prime}(s) \mathbf{N}_{1}(s)+\left(2 k_{1}^{\prime}(s) k_{2}(s)+\right.$ $\left.\left.k_{1}(s) k_{2}^{\prime}(s)\right) \mathbf{N}_{2}(s), \mp \mathbf{N}_{2}(\mathbf{s})\right\rangle=2 k_{1}^{\prime}(s) k_{2}(s)+k_{1}(s) k_{2}^{\prime}(s)=$ $k_{1}(s) k_{2}^{\prime}(s)$. Since $k_{1}(s) \neq 0$, we get that $h_{1 v}^{(3)}(s)=0$ is equivalent to condition $k_{2}^{\prime}(s)=0$. The assertion (4) follows.

(B) Using the same computation as the proof of $(\mathrm{A})$, we can get $(B)$.
(C) (1) Since $h_{3 v}(s)=\left\langle\boldsymbol{\zeta}_{1}(s), \mathbf{v}\right\rangle=\mathbf{0}$, we have that there are real numbers $\lambda$ and $\mu$ such that $\mathbf{v}=\lambda \mathbf{B}(s)+\mu \zeta_{2}(s)$. By the condition that $\mathbf{v} \in S^{2}$, we get $\lambda^{2}+\mu^{2}=1$. The converse direction also holds.

(2) Since $h_{3 v}^{\prime}(s)=\left\langle-\epsilon_{1}(s) \mathbf{B}(s), \mathbf{v}\right\rangle=0$, by the conditions of assertion (2) and $\epsilon_{1}(s) \neq 0$, we have that $\epsilon_{1}(s) \lambda=0$. It follows from the fact that $\epsilon_{1}(s) \lambda=0$ and $\epsilon_{1}(s) \neq 0$ that this is equivalent to condition $\lambda=0$. Therefore, we have $\mathbf{v}= \pm \zeta_{2}(s)$. The converse direction also holds.

(3) Since $h_{3 v}^{\prime \prime}(s)=\left\langle-\epsilon_{1}^{\prime}(s) \mathbf{B}(s)+\epsilon_{1}^{2}(s) \zeta_{1}(s)+\right.$ $\left.\epsilon_{1} \epsilon_{2}(s) \zeta_{2}(s), \mathbf{v}\right\rangle=0$, we have $\epsilon_{1}(s) \epsilon_{2}(s)=0$, by the conditions of assertion (3) and $\epsilon_{1}(s) \neq 0$ that this is equivalent to condition $\epsilon_{2}(s)=0$. Therefore, we have $\mathbf{v}= \pm \zeta_{2}(s)$ and $\epsilon_{2}(s)=0$. The converse direction also holds.

(4) Since $h_{3 v}^{(3)}(s)=\left\langle\left(\epsilon_{1}^{\prime}(s) \epsilon_{1}(s)\right) \zeta_{1}(s)+\epsilon_{1}(s) \epsilon_{2}^{\prime}(s) \zeta_{2}(s)-\right.$ $\left.\left(\epsilon_{1}^{3}(s)+\epsilon_{1}(s) \epsilon_{2}(s)^{2}-\epsilon_{1}^{\prime \prime}(s)\right) \mathbf{B}(s), \mathbf{v}\right\rangle=0$, by the conditions of assertion (4) and $\epsilon_{1}(s) \neq 0$, we have $\epsilon_{1}(s) \epsilon_{2}^{\prime}(s)=0$. This is equivalent to condition $\epsilon_{2}^{\prime}(s)=0$. Therefore, we have $\mathbf{v}=$ $\pm \zeta_{2}(s)$ and $\epsilon_{2}(s)=\epsilon_{2}^{\prime}(s)=0$. The converse direction also holds.

(D) Using the same computation as the proof of $(\mathrm{C})$, we can get (D).

Proposition 3. Let $\gamma: I \rightarrow \mathbb{E}^{3}$ be a regular unit speed curve. One has the following claims.

(1) Suppose $k_{1}(s) \neq 0$. Then, $k_{2}(s)=0$ if and only if each of $\mathbf{v}=\mp \mathbf{N}_{2}(s)$ is a constant vector.

(2) Suppose $k_{2}(s) \neq 0$. Then, $k_{1}(s)=0$ if and only if each of $\mathbf{v}=\mp \mathbf{N}_{1}(s)$ is a constant vector.

(3) Suppose $\epsilon_{1}(s) \neq 0$. Then, $\epsilon_{2}(s)=0$ if and only if each of $\mathbf{v}=\mp \boldsymbol{\zeta}_{2}(s)$ is a constant vector.

(4) Suppose $\epsilon_{2}(s) \neq 0$. Then, $\epsilon_{1}(s)=0$ if and only if each of $\mathbf{v}=\mp \zeta_{1}(s)$ is a constant vector.

Proof. (1) Suppose $k_{1}(s) \neq 0$. By straightforward calculations, we have

$$
\mathbf{v}^{\prime}(s)= \pm k_{2}(s) \mathbf{T}(s) .
$$

Thus, $\mathbf{v}^{\prime}(s) \equiv 0$ if and only if $k_{2}(s)=0$.

(2) Using the same computation as the proof of (1), we can get (2).

(3) Suppose $\epsilon_{1}(s) \neq 0$. By straightforward calculations, we have

$$
\mathbf{v}^{\prime}(s)= \pm \epsilon_{2}(s) \mathbf{B}(s) \text {. }
$$

Thus, $\mathbf{v}^{\prime}(s) \equiv 0$ if and only if $\epsilon_{2}(s)=0$.

(4) Using the same computation as the proof of (3), we can get (4).

\section{Versal Unfolding and Proof of the Main Result}

In this section, we use some general results on the singularity theory for families of function germs. Detailed descriptions can be found in the book [16]. Let $F:\left(\mathbb{R} \times \mathbb{R}^{r},\left(s_{0}, x_{0}\right)\right) \rightarrow$ 
$\mathbb{R}$ be a function germ. We call $F$ an $r$-parameter unfolding of $f(s)$, where $f(s)=F\left(s, x_{0}\right)$. We say that $f(s)$ have $A_{k^{-}}$ singularity at $s_{0}$ if $f^{(p)}\left(s_{0}\right)=0$ for all $1 \leq p \leq k$, and $f^{(k+1)}\left(s_{0}\right) \neq 0$. We also say that $f(s)$ has $A_{\geq k}$-singularity at $s_{0}$ if $f^{(p)}\left(s_{0}\right)=0$ for all $1 \leq p \leq k$. Let $F$ be an unfolding of $f(s)$ and let $f(s)$ has $A_{k}$-singularity $(k \geq 1)$ at $s_{0}$. We denote the $(k-1)$-jet of the partial derivative $\partial F / \partial x_{i}$ at $s_{0}$ by $j^{(k-1)}\left(\left(\partial F / \partial x_{i}\right)\left(x, s_{0}\right)\right)\left(s_{0}\right)=\sum_{j=1}^{k-1} a_{j i} s^{j}$ for $i=1, \ldots, r$. Then, $F$ is called a versal unfolding if the $k \times r$ matrix of coefficients $\left(a_{0 i}, a_{j i}\right)$ has rank $k,(k \leq r)$, where $a_{0 i}=\left(\partial F / \partial x_{i}\right)\left(x_{0}, s_{0}\right)$. We now introduce an important set concerning the unfolding. The discriminant set of $F$ is the set

$$
\mathfrak{D}_{F}=\left\{x \in \mathbb{R}^{r} \mid F(s, x)=\frac{\partial F}{\partial s}(s, x)=0\right\} .
$$

Then, we have the following well-known result. (cf. [16].)

Theorem 4. Let $F:\left(\mathbb{R} \times \mathbb{R}^{r},\left(s_{0}, x_{0}\right)\right) \rightarrow \mathbb{R}$ be an $r$-parameter unfolding of $f(s)$ which has the $A_{k}$ singularity at $s_{0}$.

Suppose that $F$ is a $(p)$ versal unfolding. Then, one has the following.

(a) If $k=1$, then $\mathfrak{D}_{F}$ is locally diffeomorphic to $0 \times \mathbb{R}^{r-1}$.

(b) If $k=2$, then $\mathfrak{D}_{F}$ is locally diffeomorphic to $C \times \mathbb{R}^{r-2}$.

By Proposition 2 and the definition of discriminant set, we have the following proposition.

Proposition 5. (1) The discriminant sets of $H_{1}$ and $H_{2}$ are, respectively,

$$
\begin{aligned}
& \mathfrak{D}_{H_{1}}=\left\{\mathbf{v}=\mp \mathbf{N}_{2}(s) \mid s \in I\right\}, \\
& \mathfrak{D}_{H_{2}}=\left\{\mathbf{v}=\mp \mathbf{N}_{1}(s) \mid s \in I\right\} .
\end{aligned}
$$

(2) The discriminant sets of $\mathrm{H}_{3}$ and $\mathrm{H}_{4}$ are, respectively,

$$
\begin{aligned}
& \mathfrak{D}_{H_{3}}=\left\{\mathbf{v}=\mp \zeta_{2}(s) \mid s \in I\right\}, \\
& \mathfrak{D}_{H_{4}}=\left\{\mathbf{v}=\mp \zeta_{1}(s) \mid s \in I\right\} .
\end{aligned}
$$

For the Bishop normal indicatrix height functions, we can consider the following propositions.

Proposition 6. (1) If $h_{1 v_{0}}(s)$ has $A_{k}$-singularity $(k=1,2)$ at $s_{0}$, then $H_{1}(s, \mathbf{v})$ is a $(p)$ versal unfolding of $h_{1 v_{0}}(s)$.

(2) If $h_{2 v_{0}}(s)$ has $A_{k}$-singularity $(k=1,2)$ at $s_{0}$, then $H_{2}(s, \mathbf{v})$ is a $(p)$ versal unfolding of $h_{2 v_{0}}(s)$.

(3) If $h_{3 v_{0}}(s)$ has $A_{k}$-singularity $(k=1,2)$ at $s_{0}$, then $H_{3}(s, \mathbf{v})$ is a $(p)$ versal unfolding of $h_{3 v_{0}}(s)$.

(4) If $h_{4 v_{0}}(s)$ has $A_{k}$-singularity $(k=1,2)$ at $s_{0}$, then $H_{4}(s, \mathbf{v})$ is a $(p)$ versal unfolding of $h_{4 v_{0}}(s)$.

Proof. (1) We denote that

$$
\begin{gathered}
\mathbf{N}_{1}(s)=\left(n_{11}(s), n_{12}(s), n_{13}(s)\right), \\
\mathbf{v}=\left(v_{1}, v_{2}, \pm \sqrt{1-v_{1}^{2}-v_{2}^{2}}\right) .
\end{gathered}
$$

Under this notation, we have that

$$
H_{1}(s, \mathbf{v})=n_{11}(s) v_{1}+n_{12}(s) v_{2} \pm n_{13}(s) \sqrt{1-v_{1}^{2}-v_{2}^{2}} .
$$

Thus, we have that

$$
\frac{\partial H_{1}}{\partial v_{i}}=n_{1 i}(s) \mp \frac{v_{i} n_{13}(s)}{\sqrt{1-v_{1}^{2}-v_{2}^{2}}}, \quad i=1,2 .
$$

We also have that

$$
\begin{gathered}
\frac{\partial}{\partial s} \frac{\partial H_{1}}{\partial v_{i}}=n_{1 i}^{\prime}(s) \mp \frac{v_{i} n_{13}^{\prime}(s)}{\sqrt{1-v_{1}^{2}-v_{2}^{2}}}, \quad i=1,2, \\
\frac{\partial^{2}}{\partial s^{2}} \frac{\partial H_{1}}{\partial v_{i}}=n_{1 i}^{\prime \prime}(s) \mp \frac{v_{i} n_{13}^{\prime \prime}(s)}{\sqrt{1-v_{1}^{2}-v_{2}^{2}}}, \quad i=1,2 .
\end{gathered}
$$

Therefore, the 2 -jet of $\left(\partial H_{1} / \partial v_{i}\right)(s, v)(i=1,2)$ at $s_{0}$ is given by

$$
\begin{aligned}
j^{2}\left(\frac{\partial H_{1}}{\partial v_{i}}\left(s, v_{0}\right)\right)\left(s_{0}\right) & =\frac{\partial}{\partial s} \frac{\partial H_{1}}{\partial v_{i}}\left(s-s_{0}\right)+\frac{1}{2} \frac{\partial^{2}}{\partial s^{2}} \frac{\partial H_{1}}{\partial v_{i}}\left(s-s_{0}\right)^{2} \\
& =a_{1 i}\left(s-s_{0}\right)+\frac{1}{2} a_{2 i}\left(s-s_{0}\right)^{2}
\end{aligned}
$$

It is enough to show that the rank of the matrix $A$ is 2 , where

$$
A=\left(\begin{array}{cc}
n_{11}^{\prime}(s) \mp \frac{v_{1} n_{13}^{\prime}(s)}{\sqrt{1-v_{1}^{2}-v_{2}^{2}}} & n_{12}^{\prime}(s) \mp \frac{v_{2} n_{13}^{\prime}(s)}{\sqrt{1-v_{1}^{2}-v_{2}^{2}}} \\
n_{11}^{\prime \prime}(s) \mp \frac{v_{1} n_{13}^{\prime \prime}(s)}{\sqrt{1-v_{1}^{2}-v_{2}^{2}}} & n_{12}^{\prime \prime}(s) \mp \frac{v_{2} n_{13}^{\prime \prime}(s)}{\sqrt{1-v_{1}^{2}-v_{2}^{2}}}
\end{array}\right) .
$$

Denote that $v_{3}= \pm \sqrt{1-v_{1}^{2}-v_{2}^{2}}$. Then, we have

$$
\begin{aligned}
\operatorname{det} A= & \left(n_{12}^{\prime \prime} n_{11}^{\prime}-n_{12}^{\prime} n_{11}^{\prime \prime}\right)-\frac{v_{1}}{v_{3}}\left(n_{12}^{\prime \prime} n_{13}^{\prime}-n_{12}^{\prime} n_{13}^{\prime \prime}\right) \\
& -\frac{v_{2}}{v_{3}}\left(n_{13}^{\prime \prime} n_{11}^{\prime}-n_{13}^{\prime} n_{11}^{\prime \prime}\right) \\
= & \frac{1}{v_{3}}\left\langle\mathbf{v}, \mathbf{N}_{1}^{\prime}(s) \wedge \mathbf{N}_{1}^{\prime \prime}(s)\right\rangle \\
= & \frac{1}{v_{3}}\left\langle\mathbf{v},-k_{1}(s) \mathbf{T}(s) \wedge-k_{1}^{\prime}(s) \mathbf{T}(s)\right. \\
= & \frac{1}{v_{3}}\left\langle\mathbf{v}, k_{1}^{3}(s) \mathbf{N}_{2}(s)-k_{1}^{2}(s) k_{2}(s) \mathbf{N}_{1}(s)\right\rangle \\
= & \frac{1}{v_{3}}\left\langle\mp \mathbf{N}_{2}(s), k_{1}^{3}(s) \mathbf{N}_{2}(s)-k_{1}^{2}(s) k_{2}(s) \mathbf{N}_{1}(s)\right\rangle \\
= & \mp \frac{1}{v_{3}} k_{1}^{3}(s) \neq 0 .
\end{aligned}
$$


Note that $\mathbf{v} \in \mathfrak{D}_{H_{1}}$ is a singular point, where

$$
\mathbf{v}=\mp \mathbf{N}_{2}(s) \text {. }
$$

This completes the proof.

(2) Using the same computation as the proof of (1), we can get (2).

(3) We denote that

$$
\begin{gathered}
\zeta_{1}(s)=\left(\zeta_{11}(s), \zeta_{12}(s), \zeta_{13}(s)\right), \\
\mathbf{v}=\left(v_{1}, v_{2}, \pm \sqrt{1-v_{1}^{2}-v_{2}^{2}}\right) .
\end{gathered}
$$

Under this notation, we have

$$
H_{3}(s, \mathbf{v})=\zeta_{11}(s) v_{1}+\zeta_{12}(s) v_{2} \pm \zeta_{13}(s) \sqrt{1-v_{1}^{2}-v_{2}^{2}}
$$

Thus, we have

$$
\frac{\partial H_{3}}{\partial v_{i}}=\zeta_{1 i}(s) \mp \frac{v_{i} \zeta_{13}(s)}{\sqrt{1-v_{1}^{2}-v_{2}^{2}}}, \quad i=1,2
$$

We also have

$$
\begin{aligned}
& \frac{\partial}{\partial s} \frac{\partial H_{3}}{\partial v_{i}}=\zeta_{1 i}^{\prime}(s) \mp \frac{v_{i} \zeta_{13}^{\prime}(s)}{\sqrt{1-v_{1}^{2}-v_{2}^{2}}}, \quad i=1,2, \\
& \frac{\partial^{2}}{\partial s^{2}} \frac{\partial H_{3}}{\partial v_{i}}=\zeta_{1 i}^{\prime \prime}(s) \mp \frac{v_{i} \zeta_{13}^{\prime \prime}(s)}{\sqrt{1-v_{1}^{2}-v_{2}^{2}}}, \quad i=1,2 .
\end{aligned}
$$

Therefore, the 2 -jet of $\left(\partial H_{3} / \partial v_{i}\right)(s, v)(i=1,2)$ at $s_{0}$ is given by

$$
\begin{aligned}
j^{2}\left(\frac{\partial H_{3}}{\partial v_{i}}\left(s, v_{0}\right)\right)\left(s_{0}\right) & =\frac{\partial}{\partial s} \frac{\partial H_{3}}{\partial v_{i}}\left(s-s_{0}\right)+\frac{1}{2} \frac{\partial^{2}}{\partial s^{2}} \frac{\partial H_{3}}{\partial v_{i}}\left(s-s_{0}\right)^{2} \\
& =a_{1 i}\left(s-s_{0}\right)+\frac{1}{2} a_{2 i}\left(s-s_{0}\right)^{2} .
\end{aligned}
$$

It is enough to show that the rank of the matrix $B$ is 2 , where

$$
B=\left(\begin{array}{c}
\zeta_{11}^{\prime}(s) \mp \frac{v_{1} \zeta_{13}^{\prime}(s)}{\sqrt{1-v_{1}^{2}-v_{2}^{2}}} \zeta_{12}^{\prime}(s) \mp \frac{v_{2} \zeta_{13}^{\prime}(s)}{\sqrt{1-v_{1}^{2}-v_{2}^{2}}} \\
\zeta_{11}^{\prime \prime}(s) \mp \frac{v_{1} \zeta_{13}^{\prime \prime}(s)}{\sqrt{1-v_{1}^{2}-v_{2}^{2}}} \zeta_{12}^{\prime \prime}(s) \mp \frac{v_{2} \zeta_{13}^{\prime \prime}(s)}{\sqrt{1-v_{1}^{2}-v_{2}^{2}}}
\end{array}\right) .
$$

Denote that $v_{3}= \pm \sqrt{1-v_{1}^{2}-v_{2}^{2}}$. Then, we have

$$
\begin{aligned}
& \operatorname{det} B=\left(\zeta_{12}^{\prime \prime} \zeta_{11}^{\prime}-\zeta_{12}^{\prime} \zeta_{11}^{\prime \prime}\right)-\frac{v_{1}}{v_{3}}\left(\zeta_{12}^{\prime \prime} \zeta_{13}^{\prime}-\zeta_{12}^{\prime} \zeta_{13}^{\prime \prime}\right) \\
&-\frac{v_{2}}{v_{3}}\left(\zeta_{13}^{\prime \prime} \zeta_{11}^{\prime}-\zeta_{13}^{\prime} \zeta_{11}^{\prime \prime}\right) \\
&= \frac{1}{v_{3}}\left\langle\mathbf{v}, \zeta_{1}^{\prime}(s) \wedge \zeta_{1}^{\prime \prime}(s)\right\rangle \\
&= \frac{1}{v_{3}}\left\langle\mathbf{v},\left(-\epsilon_{1}(s) \mathbf{B}(s)\right)\right. \\
& \quad \wedge\left(-\epsilon_{1}^{\prime}(s) \mathbf{B}(s)-\epsilon_{1}^{2}(s) \zeta_{1}(s)\right. \\
&=\frac{1}{v_{3}}\left\langle\mathbf{v}, \epsilon_{1}^{3}(s) \zeta_{2}(s)-\epsilon_{1}^{3}(s) \epsilon_{2}(s) \zeta_{1}(s)\right\rangle \\
&=\frac{1}{v_{3}}\left\langle\mp \zeta_{2}(s), \epsilon_{1}^{3}(s) \zeta_{2}(s)-\epsilon_{1}^{3}(s) \epsilon_{2}(s) \zeta_{1}(s)\right\rangle \\
&=\mp \frac{1}{v_{3}} \epsilon_{1}^{3}(s) \neq 0 .
\end{aligned}
$$

Note that $\mathbf{v} \in \mathfrak{D}_{\mathrm{H}_{3}}$ is a singular point, where

$$
\mathbf{v}=\mp \zeta_{2}(s) .
$$

This completes the proof.

(4) Using the same computation as the proof of (3), we can get (4).

Proof of Theorem 1. (1) By Proposition 5, the discriminant set $\mathfrak{D}_{\mathrm{H}_{2}}$ of $\mathrm{H}_{2}$ is

$$
\mathfrak{D}_{H_{2}}=\left\{\mathbf{v}=\mp \mathbf{N}_{1}(s) \mid s \in I\right\} \text {. }
$$

The assertion (1) of Theorem 1 follows from Proposition 6 and Theorem 4.

(2) By Proposition 5, the discriminant set $\mathfrak{D}_{H_{1}}$ of $H_{1}$ is

$$
\mathfrak{D}_{H_{1}}=\left\{\mathbf{v}=\mp \mathbf{N}_{2}(s) \mid s \in I\right\} .
$$

The assertion (2) of Theorem 1 follows from Proposition 6 and Theorem 4.

(3) By Proposition 5, the discriminant set $\mathfrak{D}_{H_{4}}$ of $H_{4}$ is

$$
\mathfrak{D}_{H_{4}}=\left\{\mathbf{v}=\mp \zeta_{1}(s) \mid s \in I\right\} .
$$

The assertion (3) of Theorem 1 follows from Proposition 6 and Theorem 4.

(4) By Proposition 5, the discriminant set $\mathfrak{D}_{H_{3}}$ of $\mathrm{H}_{3}$ is

$$
\mathfrak{D}_{H_{3}}=\left\{\mathbf{v}=\mp \zeta_{2}(s) \mid s \in I\right\} .
$$

The assertion (4) of Theorem 1 follows from Proposition 6 and Theorem 4. 


\section{Generic Properties}

In this section, we consider generic properties of regular curves in $\mathbb{E}^{3}$. The main tool is a kind of transversality theorem. Let $\operatorname{Emb}_{B}\left(I, \mathbb{E}^{3}\right)$ be the space of embeddings $\gamma$ : $I \rightarrow \mathbb{E}^{3}$ with $k_{i}(s) \neq 0$ or $\epsilon_{j}(s) \neq 0$ equipped with Whitney $C^{\infty}$-topology. Here $i=1,2$ and $j=3,4$. We also consider the function $\mathscr{H}_{k}: \mathbb{E}^{3} \times S^{2} \rightarrow \mathbb{R}$ defined by $\mathscr{H}_{k}(\mathbf{u}, \mathbf{v})=\langle\mathbf{u}, \mathbf{v}\rangle$. Here $k=1,2,3,4$. We claim that $\mathscr{H}_{k \mathbf{v}}$ is a submersion for any $\mathbf{v} \in S^{2}$, where $h_{k v}(\mathbf{u})=\mathscr{H}_{k}(\mathbf{u}, \mathbf{v})$. For any $\gamma \in \operatorname{Emb}_{B}\left(I, \mathbb{E}^{3}\right)$, we have $H_{k}=\mathscr{H}_{k} \circ\left(\gamma \times i d_{S^{2}}\right)$. We also have the $\ell$-jet extension $j_{1}^{\ell} H_{k}: I \times S^{2} \rightarrow J^{\ell}(I, \mathbb{R})$ defined by $j_{1}^{\ell} H_{k}(s, \mathbf{v})=j^{\ell} h_{k v}(s)$. We consider the trivialization $J^{\ell}(I, \mathbb{R}) \equiv I \times \mathbb{R} \times J^{\ell}(1,1)$. For any submanifold $Q \subset J^{\ell}(1,1)$, we denote that $\widetilde{Q}=I \times\{0\} \times Q$. It is evident that both $j_{1}^{\ell} H_{k}$ is a submersions and $\widetilde{Q}$ is an immersed submanifold of $j^{\ell}(I, \mathbb{R})$. Then $j_{1}^{\ell} H_{k}$ is transversal to $\widetilde{Q}$. We have the following proposition as a corollary of Lemma 6 in Wassermann [17].

Proposition 7. Let $Q$ be a submanifold of $J^{\ell}(1,1)$. Then the set

$$
T_{Q}=\left\{\gamma \in \operatorname{Emb}_{B}\left(I, \mathbb{E}^{3}\right) \mid j^{\ell} H_{k} \text { is transversal to } \widetilde{Q}\right\}
$$

is a residual subset of $\operatorname{Emb}\left(I, \mathbb{E}^{3}\right)$. If $Q$ is a closed subset, then $T_{\mathrm{Q}}$ is open.

Let $f:(\mathbb{R}, 0) \rightarrow(\mathbb{R}, 0)$ be a function germ which has an $A_{k}$-singularity at 0 . It is well known that there exists a diffeomorphism germ $\phi:(\mathbb{R}, 0) \rightarrow(\mathbb{R}, 0)$ such that $f \circ \phi= \pm s^{k+1}$. This is the classification of $A_{k}$-singularities. For any $z=j^{l} f(0)$ in $J^{\ell}(1,1)$, we have the orbit $L^{l}(z)$ given by the action of the Lie group of $l$-jet diffeomorphism germs. If $f$ has an $A_{k}$-singularity, then the codimension of the orbit is $k$. There is another characterization of versal unfolding as follows [15].

Proposition 8. Let $F:\left(\mathbb{R} \times \mathbb{R}^{r}, 0\right) \rightarrow(\mathbb{R}, 0)$ be an $r$ parameter unfolding of $f:(\mathbb{R}, 0) \rightarrow(\mathbb{R}, 0)$ which has an $A_{k}$-singularity at 0 . Then $F$ is a versal unfolding if and only if $j_{1}^{l} F$ is transversal to the orbit $L^{l}\left(\widetilde{j^{l} f(0)}\right)$ for $l \geq k+1$. Here, $j_{1}^{l}\left(\mathbb{R} \times \mathbb{R}^{r}, 0\right) \rightarrow J^{\ell}(\mathbb{R}, \mathbb{R})$ is the l-jet extension of $F$ given by $j_{1}^{l} F(s, \mathbf{x})=j^{l} F_{x}(s)$.

The generic classification theorem is given as follows.

Theorem 9. There exists an open and dense subset $\mathcal{O} C$ $\operatorname{Emb}_{B}\left(I, \mathbb{E}^{3}\right)$ such that for any $\gamma \in \mathcal{O}$, the Bishop spherical images $\mathbf{N}_{i}(s), i=1,2$ and the type-2 Bishop spherical images $\zeta_{j}(s), j=3,4$ of $\gamma(s)$, are locally diffeomorphic to the ordinary cusp at any singular point.

\section{Examples}

As applications and illustration of the main results (Theorem 1), we give two examples in this section.

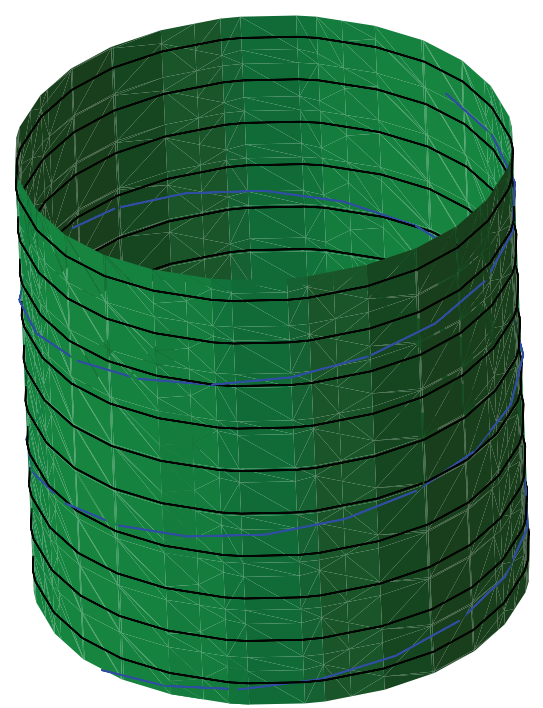

Figure 2: Curve.

Example 1. Let $\gamma(s)$ be a unit speed curve of $\mathbb{E}^{3}$ defined by

$$
\gamma(s)=\left(3 \cos \frac{1}{5} s, 3 \sin \frac{1}{5} s, \frac{4}{5} s\right)
$$

with respect to a arclength parameter $s$, where $s \in[0,50 \pi]$; see Figure 2.

The curvature and torsion of this curve are, respectively, as follows:

$$
\begin{aligned}
& k(s)=\frac{3}{25}, \\
& \tau(s)=\frac{4}{25} .
\end{aligned}
$$

Using the Bishop curvature equations (5) and (8), we obtain the following:

$$
\begin{aligned}
k_{1}(s) & =\frac{3}{25} \cos \left(\frac{4}{25} s\right), \\
k_{2}(s) & =\frac{3}{25} \sin \left(\frac{4}{25} s\right), \\
\epsilon_{1}(s) & =-\frac{4}{25} \cos \left(\frac{3}{25} s\right), \\
\epsilon_{2}(s) & =-\frac{4}{25} \sin \left(\frac{3}{25} s\right) .
\end{aligned}
$$

Using (3) and (7), we obtain the vector parametric equations of the Bishop spherical images $\mathbf{N}_{1}(s), \mathbf{N}_{2}(s)$ and the type-2 Bishop spherical images $\zeta_{1}(s), \zeta_{2}(s)$ as follows:

$$
\begin{aligned}
\mathbf{N}_{1}(s)=( & -\cos \left(\frac{4}{25} s\right) \cos (15 s) \\
& -\frac{4}{5} \sin \left(\frac{4}{25} s\right) \sin \left(\frac{1}{5} s\right), \\
& -\cos \left(\frac{4}{25} s\right) \sin (15 s)
\end{aligned}
$$




$$
\begin{aligned}
&\left.+\frac{4}{5} \sin \left(\frac{4}{25} s\right) \cos \left(\frac{1}{5} s\right),-\frac{3}{5} \sin \left(\frac{4}{25} s\right)\right), \\
& \mathbf{N}_{2}(s)=( \sin \left(\frac{4}{25} s\right) \cos (15 s) \\
&+\frac{4}{5} \cos \left(\frac{4}{25} s\right) \sin \left(\frac{1}{5} s\right), \\
&-\sin \left(\frac{4}{25} s\right) \sin (15 s) \\
&\left.-\frac{4}{5} \cos \left(\frac{4}{25} s\right) \cos \left(\frac{1}{5} s\right), \frac{3}{5} \cos \left(\frac{4}{25} s\right)\right), \\
& \zeta_{1}(s)=(-\frac{3}{5} \sin \left(\frac{3}{25} s\right) \sin (15 s)-\cos \left(\frac{3}{25} s\right) \cos \left(\frac{1}{5} s\right), \\
& \frac{3}{5} \sin \left(\frac{3}{25} s\right) \cos (15 s) \\
&\left.-\cos \left(\frac{3}{25} s\right) \sin \left(\frac{1}{5} s\right), \frac{4}{5} \sin \left(\frac{3}{25} s\right)\right), \\
& \zeta_{2}(s)=\left(\frac{3}{5} \cos \left(\frac{3}{25} s\right) \sin (15 s)-\sin \left(\frac{3}{25} s\right) \cos \left(\frac{1}{5} s\right),\right. \\
& \quad-\frac{3}{5} \cos \left(\frac{3}{25} s\right) \cos (15 s) \\
&\left.\quad-\sin \left(\frac{3}{25} s\right) \sin \left(\frac{1}{5} s\right),-\frac{4}{5} \cos \left(\frac{3}{25} s\right)\right) .
\end{aligned}
$$

We see that $k_{1}(s)=0$ gives some real roots $s=(25 / 4) k \pi+$ $(25 / 8) \pi$, and $k_{1}^{\prime}(s)=0$ gives some real roots $s=(25 / 4) k \pi$. On the other hand, equation $k_{2}(s)=0$ gives some real roots $s=(25 / 4) k \pi$ and $k_{2}^{\prime}(s)=0$ gives some real roots $s=(25 / 4) k \pi+(25 / 8) \pi$, where $k=0,1, \ldots, 7$. For the case that $s=(25 / 4) k \pi+(25 / 8) \pi$, we have that $k_{1}(s)=0$ and $k_{1}^{\prime}(s) \neq 0$. Hence, we have that the first Bishop spherical image $\mathbf{N}_{1}(s)$ is locally diffeomorphic to cusp and it has eight cusps; see Figure 3. We can also get that the second Bishop spherical image $\mathbf{N}_{2}(s)$ is locally diffeomorphic to cusp at $s=(25 / 4) k \pi$ and it also has eight cusps; see Figure 4 . For the case that $s=(25 / 3) k \pi+(25 / 6) \pi$, where $k=0, \ldots, 5$, we have $\epsilon_{1}(s)=0$ and $\epsilon_{1}^{\prime}(s) \neq 0$. Hence, we have that the first type-2 Bishop spherical image $\zeta_{1}(s)$ is locally diffeomorphic to cusp and it has six cusps; see Figure 5. We also can get that the second type-2 Bishop spherical image $\zeta_{2}(s)$ is locally diffeomorphic to cusp at $s=(25 / 3) k \pi$, where $k=0, \ldots, 5$, and it also has six cusps; see Figure 6.

Example 2. Let $\gamma(s)$ be a unit speed curve of $\mathbb{E}^{3}$ defined by

$$
\begin{aligned}
\gamma(s)=( & \frac{2}{5} \sin 2 s-\frac{1}{40} \sin 8 s, \\
& \left.\quad-\frac{2}{5} \cos 2 s+\frac{1}{40} \cos 8 s, \frac{4}{15} \sin 3 s\right)
\end{aligned}
$$

with respect to a arclength parameter $s$, where $s \in$ $[0.34 \pi, 0.66 \pi]$; see Figure 7 . For more details about this kind of curve, see [18].

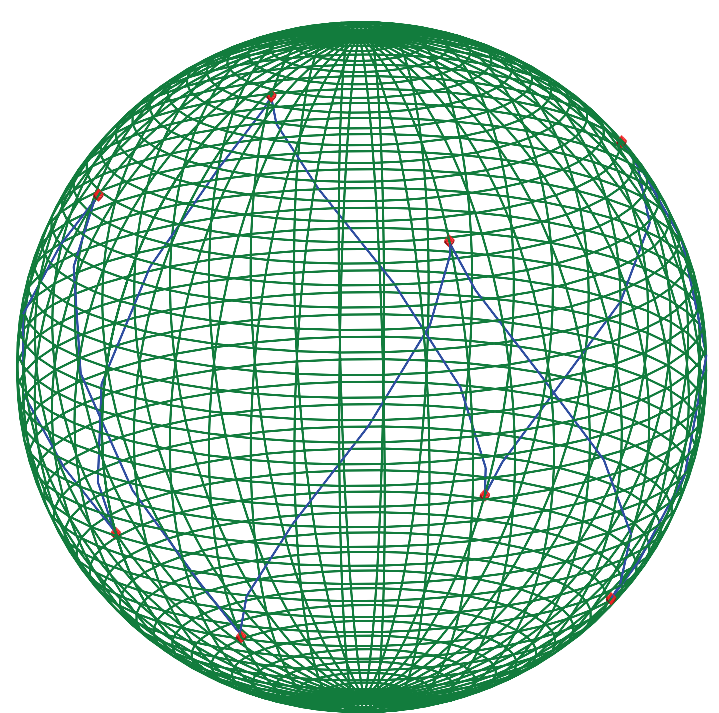

FIGURE 3: The first Bishop spherical image which has eight cusps.

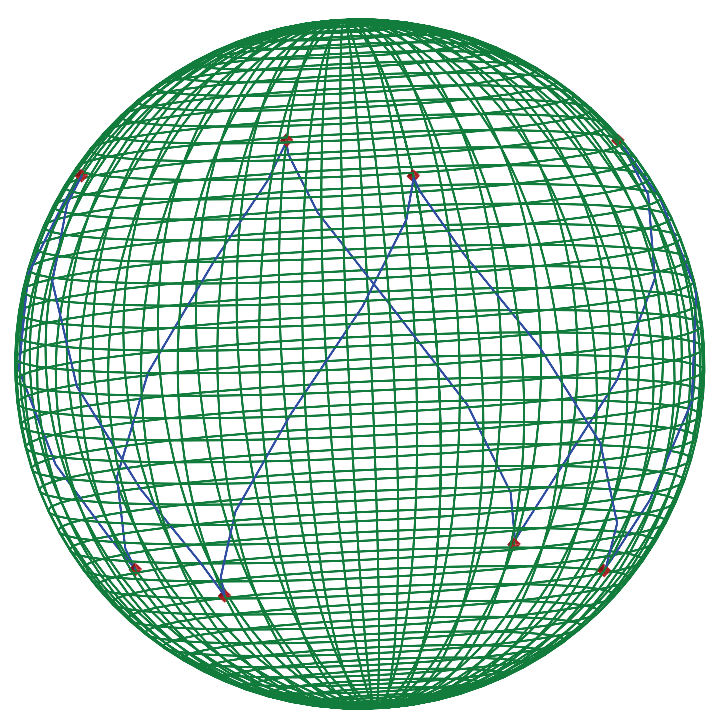

FIGURE 4: The second Bishop spherical image which has eight cusps.

The curvature and torsion of this curve are, respectively, as follows:

$$
\begin{gathered}
k(s)=-4 \sin 3 s, \\
\tau(s)=4 \cos 3 s .
\end{gathered}
$$

Using the Bishop curvature equations (5) and (8), we obtain

$$
\begin{aligned}
& k_{1}(s)=-4 \sin (3 s) \cos \left(\frac{4}{3} \sin 3 s\right), \\
& k_{2}(s)=-4 \sin (3 s) \sin \left(\frac{4}{3} \sin 3 s\right), \\
& \epsilon_{1}(s)=-4 \cos (3 s) \cos \left(\frac{4}{3} \cos 3 s\right), \\
& \epsilon_{2}(s)=-4 \cos (3 s) \sin \left(\frac{4}{3} \cos 3 s\right) .
\end{aligned}
$$




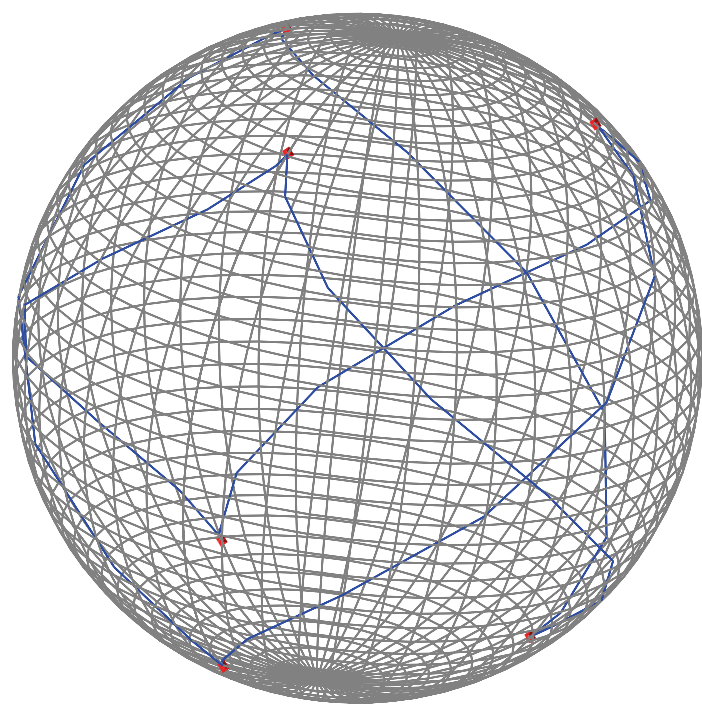

Figure 5: The first type-2 Bishop spherical image which has six cusps.

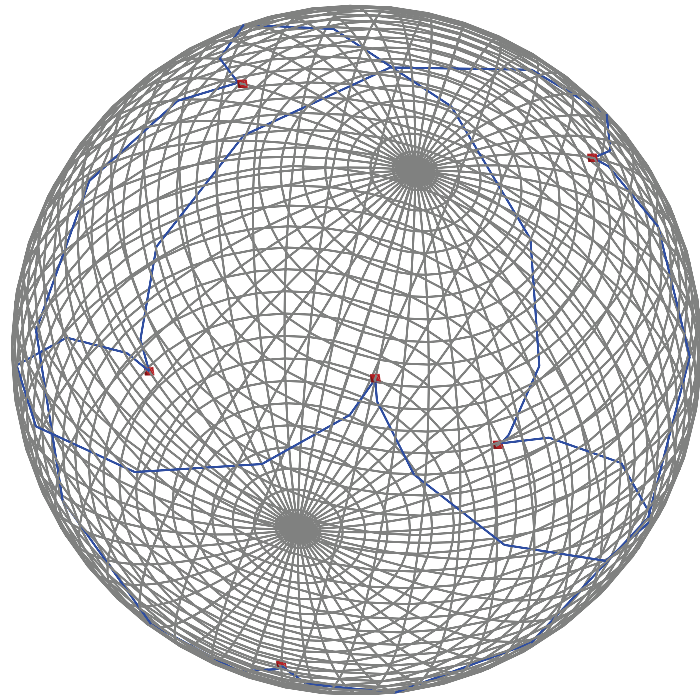

Figure 6: The second type-2 Bishop spherical image which has six cusps.

Using (3) and (7), we obtain the Bishop spherical images $\mathbf{N}_{1}(s), \mathbf{N}_{2}(s)$ and the type-2 Bishop spherical images $\zeta_{1}(s), \zeta_{2}(s)$ as follows:

$$
\begin{aligned}
\mathbf{N}_{1}(s)= & \frac{1}{\sqrt{\sin ^{2} s\left(4 \cos ^{2} s-1\right)^{2}}} \\
& \times((\sin 8 s-\sin 2 s) \\
& \times\left(\frac{2}{5} \cos \left(\frac{4}{3} \sin 3 s\right)-\frac{3}{25} \sin \left(\frac{4}{3} \sin 3 s\right) \sin 3 s\right) \\
& \left.+\frac{24}{125} \sin \left(\frac{4}{3} \sin 3 s\right) \cos 3 s(\cos 8 s-\cos 2 s)\right),
\end{aligned}
$$

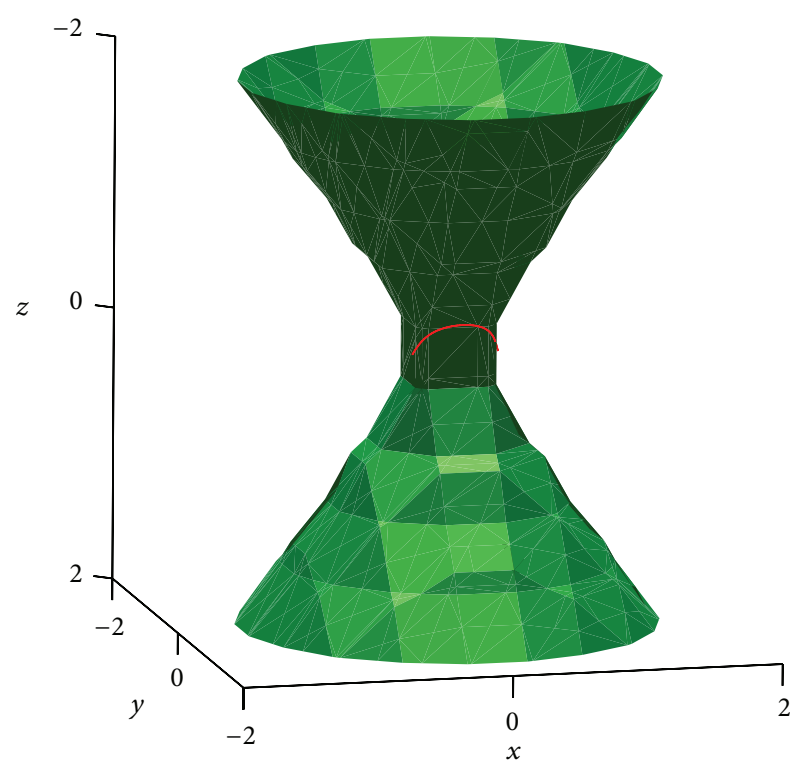

Figure 7: Curve.
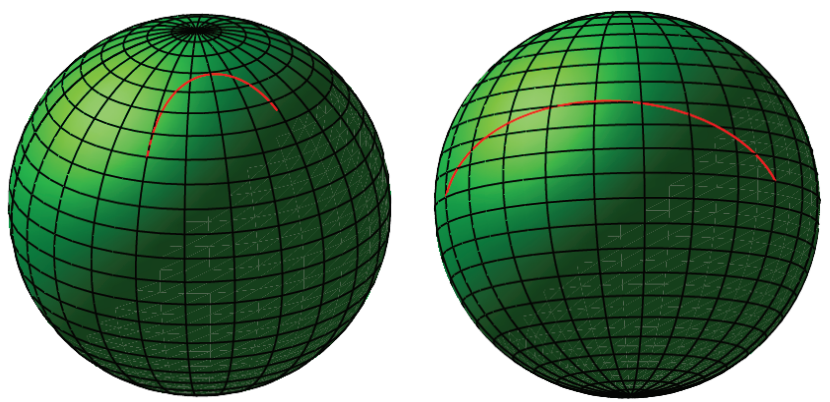

Figure 8: (a) The first Bishop spherical image. (b) The second Bishop spherical image.

$$
\begin{aligned}
& \frac{2}{5} \cos \left(\frac{4}{3} \sin 3 s\right)(\cos 2 s-\cos 8 s)-\sin \left(\frac{4}{3} \sin 3 s\right) \\
& \times\left(\frac{8}{25} \cos 3 s(\sin 8 s-\sin 2 s)\right. \\
& \left.\quad+\left(\frac{12}{25} \cos 2 s-\frac{3}{25} \cos 8 s\right) \sin 3 s\right) \\
& -\frac{3}{5} \cos \left(\frac{4}{3} \sin 3 s\right) \sin 3 s-\sin \left(\frac{4}{3} \sin 3 s\right) \\
& \times\left(\left(\frac{8}{25} \cos 2 s-\frac{2}{25} \cos 8 s\right)(\cos 2 s-\cos 8 s)\right. \\
& \mathbf{N}_{2}(s)=\frac{\left.\left.\quad+\left(\frac{8}{25} \sin 2 s-\frac{2}{25} \sin 8 s\right)(\sin 2 s-\sin 8 s)\right)\right)}{\sqrt{\sin ^{2} s\left(4 \cos ^{2} s-1\right)^{2}}} \\
& \times\left(\frac{2}{5}(\sin 8 s-\sin 2 s) \sin \left(\frac{4}{3} \sin 3 s\right)\right.
\end{aligned}
$$



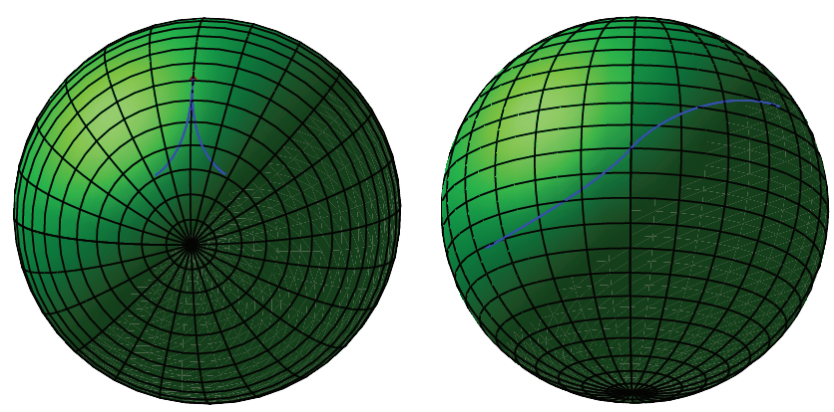

FIgure 9: (a) The first type-2 Bishop spherical image. (b) The second type-2 Bishop spherical image.

$$
\begin{aligned}
& +\cos \left(\frac{4}{3} \sin 3 s\right)\left(\frac{3}{25} \sin 8 s-\frac{12}{25} \sin 2 s\right) \sin 3 s \\
& -\frac{8}{25} \cos 3 s(\cos 2 s-\cos 8 s) \text {, } \\
& \frac{2}{5}(\cos 2 s-\cos 8 s) \sin \left(\frac{4}{3} \sin 3 s\right) \\
& +\cos \left(\frac{4}{3} \sin 3 s\right) \\
& \times\left(\frac{8}{25} \cos 3 s(\sin 8 s-\sin 2 s)\right. \\
& \left.+\left(\frac{12}{25} \cos 2 s-\frac{3}{25} \cos 8 s\right) \sin 3 s\right), \\
& \frac{-3}{5} \sin \left(\frac{4}{3} \sin 3 s\right) \sin 3 s+\cos \left(\frac{4}{3} \sin 3 s\right) \\
& \times\left(\left(\frac{8}{25} \cos 2 s-\frac{2}{25} \cos 8 s\right)(\cos 2 s-\cos 8 s)\right. \\
& \left.\left.-\left(\frac{8}{25} \sin 2 s-\frac{2}{25} \sin 8 s\right)(\sin 8 s-\sin 2 s)\right)\right) . \\
& \zeta_{1}(s)=\left(\sin \left(\frac{4}{3} \cos (3 s)\right)\left(\frac{4}{5} \cos (2 s)-\frac{1}{5} \cos (8 s)\right)+\frac{1}{4}\right. \\
& \times \frac{\cos ((4 / 3) \cos (3 s))(-(8 / 5) \sin 2 s+(8 / 5) \sin 8 s)}{\sqrt{\sin ^{2}(s)\left(4 \cos ^{2}(s)-1\right)^{2}}}, \\
& \sin \left(\frac{4}{3} \cos (3 s)\right)\left(\frac{4}{5} \sin (2 s)-\frac{1}{5} \sin (8 s)\right)+\frac{1}{4} \\
& \times \frac{\cos ((4 / 3) \cos (3 s))((8 / 5) \cos 2 s-(8 / 5) \cos 8 s)}{\sqrt{\sin ^{2}(s)\left(4 \cos ^{2}(s)-1\right)^{2}}}, \\
& \frac{4}{5} \sin \left(\frac{4}{3} \cos 3 s\right) \cos 3 s \\
& \left.-\frac{3}{5} \frac{\cos ((4 / 3) \cos 3 s) \sin 3 s}{\sqrt{\sin ^{2}(s)\left(4 \cos ^{2}(s)-1\right)^{2}}}\right),
\end{aligned}
$$

$$
\begin{aligned}
\zeta_{2}(s)=( & -\cos \left(\frac{4}{3} \cos (3 s)\right)\left(\frac{4}{5} \cos (2 s)-\frac{1}{5} \cos (8 s)\right)+\frac{1}{4} \\
& \times \frac{\sin ((4 / 3) \cos (3 s))(-(8 / 5) \sin 2 s+(8 / 5) \sin 8 s)}{\sqrt{\sin ^{2}(s)\left(4 \cos ^{2}(s)-1\right)^{2}}} \\
& -\cos \left(\frac{4}{3} \cos (3 s)\right)\left(\frac{4}{5} \sin (2 s)-\frac{1}{5} \sin (8 s)\right)+\frac{1}{4} \\
& \times \frac{\sin ((4 / 3) \cos (3 s))((8 / 5) \cos 2 s-(8 / 5) \cos 8 s)}{\sqrt{\sin ^{2}(s)\left(4 \cos ^{2}(s)-1\right)^{2}}} \\
& -\frac{4}{5} \cos \left(\frac{4}{3} \cos 3 s\right) \cos 3 s \\
& \left.-\frac{3}{5} \frac{\sin ((4 / 3) \cos 3 s) \sin 3 s}{\sqrt{\sin ^{2}(s)\left(4 \cos ^{2}(s)-1\right)^{2}}}\right)
\end{aligned}
$$

We see that $k_{1}(s)=0$ gives some real roots $s=(1 / 3) k \pi$ and $k_{1}^{\prime}(s)=0$ gives some real roots $s=(1 / 3) k \pi+(\pi / 6)$, where $k$ is an integer. Since $s \in[0.34 \pi, 0.66 \pi]$, we have that $k_{1}(s) \neq 0$ and $k_{2}(s) \neq 0$. Hence, we have that $\mathbf{N}_{1}(s)$ and $\mathbf{N}_{2}(s)$ are locally diffeomorphic to a line. The pictures of Bishop spherical images are visualized in Figure 8. For the case that $s=(1 / 2) \pi$, we have that $\epsilon_{1}(s)=0$ and $\epsilon_{1}^{\prime}(s) \neq 0$. Hence, the first type-2 Bishop spherical image $\zeta_{1}(s)$ is locally diffeomorphic to cusp; see Figure 9. We also can get that the second type-2 Bishop spherical image $\zeta_{2}(s)$ is not locally diffeomorphic to cusp; see Figure 9.

\section{Conclusions}

In this paper, we introduce the notions of Bishop normal indicatrix height functions on a space curve embedded in Euclidean 3-space. We use the Bishop Frame, the type-2 Bishop Frame, and those functions to study Bishop spherical images from the singularity viewpoint. We find that the singular points of the Bishop spherical images and Type2 Bishop spherical images correspond to the point where Bishop curvatures and type-2 Bishop curvatures vanished and their derivatives are not equal to zero. As applications of the main results, we give two examples and make them visualized by computer.

\section{Acknowledgments}

The authors would like to thank the referee for his/her valuable suggestions which improved the first version of the paper. The first author was partially supported by the Fundamental Research Funds for the Central Universities (Graduate Innovation Fund of Northeast Normal University, no. 12SSXT140), Preparatory Studies of Provincial Innovation Project of Mudanjiang Normal University, no. SY201225 and no. SY201320. The second author (Corresponding author) was partially supported by NSF of China, no. 11271063. 


\section{References}

[1] R. L. Bishop, "There is more than one way to frame a curve," The American Mathematical Monthly, vol. 82, no. 3, pp. 246-251, 1975.

[2] N. Clauvelin, W. K. Olson, and I. Tobias, "Characterization of the geometry and topology of DNA pictured as a discrete collection of atoms," Journal of Chemical Theory and Computation, vol. 8, no. 3, pp. 1092-1107, 2012.

[3] C. Y. Han, "Nonexistence of rational rotation-minimizing frames on cubic curves," Computer Aided Geometric Design, vol. 25, no. 4-5, pp. 298-304, 2008.

[4] K. Shoeemake, "Animating rotation with quaternion curves," in Proceedings of the 12th Annual Conference on Computer Graphics and Interactive Techniques, pp. 245-254, 1985.

[5] A. J. Hanson and H. Ma, "Parallel transport approach to curve framing," Indiana University 425, 1995, vol. 11.

[6] H. Liu and D. Pei, "Singularities of a space curve according to the relatively parallel adapted frame and its visualization," Mathematical Problems in Engineering, vol. 2013, Article ID 512020, 12 pages, 2013.

[7] S. Yilmaz and M. Turgut, "A new version of Bishop frame and an application to spherical images," Journal of Mathematical Analysis and Applications, vol. 371, no. 2, pp. 764-776, 2010.

[8] B. Bükcü and M. K. Karacan, "Special Bishop motion and Bishop Darboux rotation axis of the space curve," Journal of Dynamical Systems and Geometric Theories, vol. 6, no. 1, pp. 2734, 2008.

[9] B. Bükcü and M. K. Karacan, "The slant helices according to Bishop frame," International Journal of Computational and Mathematical Sciences, vol. 3, no. 2, pp. 67-70, 2009.

[10] L. Kula and Y. Yayli, "On slant helix and its spherical indicatrix," Applied Mathematics and Computation, vol. 169, no. 1, pp. 600607, 2005.

[11] S. Yılmaz, E. Özyılmaz, and M. Turgut, "New spherical indicatrices and their characterizations," Analele stiintifice ale Universitatii Ovidius Constanta, vol. 18, no. 2, pp. 337-353, 2010.

[12] L. Kula, N. Ekmekci, Y. Yaylı, and K. İlarslan, "Characterizations of slant helices in Euclidean 3-space," Turkish Journal of Mathematics, vol. 34, no. 2, pp. 261-273, 2010.

[13] M. K. Karacan and B. Bükcü, "An alternative moving frame for tubular surfaces around timelike curves in the Minkowski 3space," Balkan Journal of Geometry and its Applications, vol. 12, no. 2, pp. 73-80, 2007.

[14] D. Pei and T. Sano, "The focal developable and the binormal indicatrix of a nonlightlike curve in Minkowski 3-space," Tokyo Journal of Mathematics, vol. 23, no. 1, pp. 211-225, 2000.

[15] Z. Wang, D. Pei, L. Chen, L. Kong, and Q. Han, "Singularities of focal surfaces of null Cartan curves in Minkowski 3-space," Abstract and Applied Analysis, vol. 2012, Article ID 823809, 20 pages, 2012.

[16] J. W. Bruce and P. J. Giblin, Curves and Singularities, Cambridge University Press, Cambridge, UK, 2nd edition, 1992.

[17] G. Wassermann, "Stability of caustics," Mathematische Annalen, vol. 216, pp. 43-50, 1975.

[18] P. D. Scofield, "Curves of constant precession," The American Mathematical Monthly, vol. 102, no. 6, pp. 531-537, 1995. 


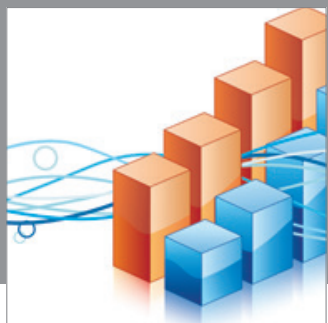

Advances in

Operations Research

mansans

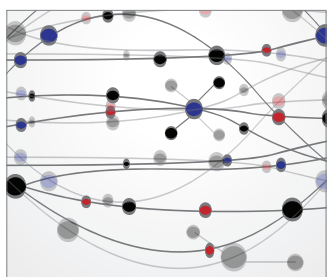

The Scientific World Journal
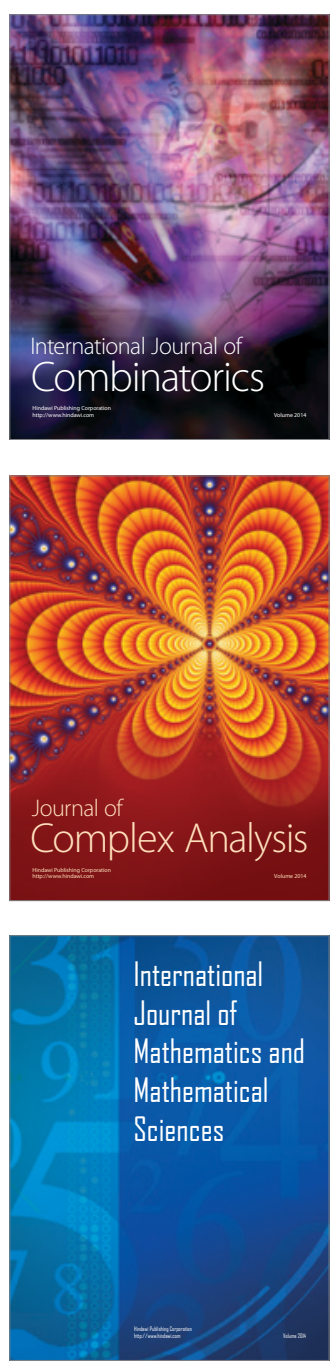
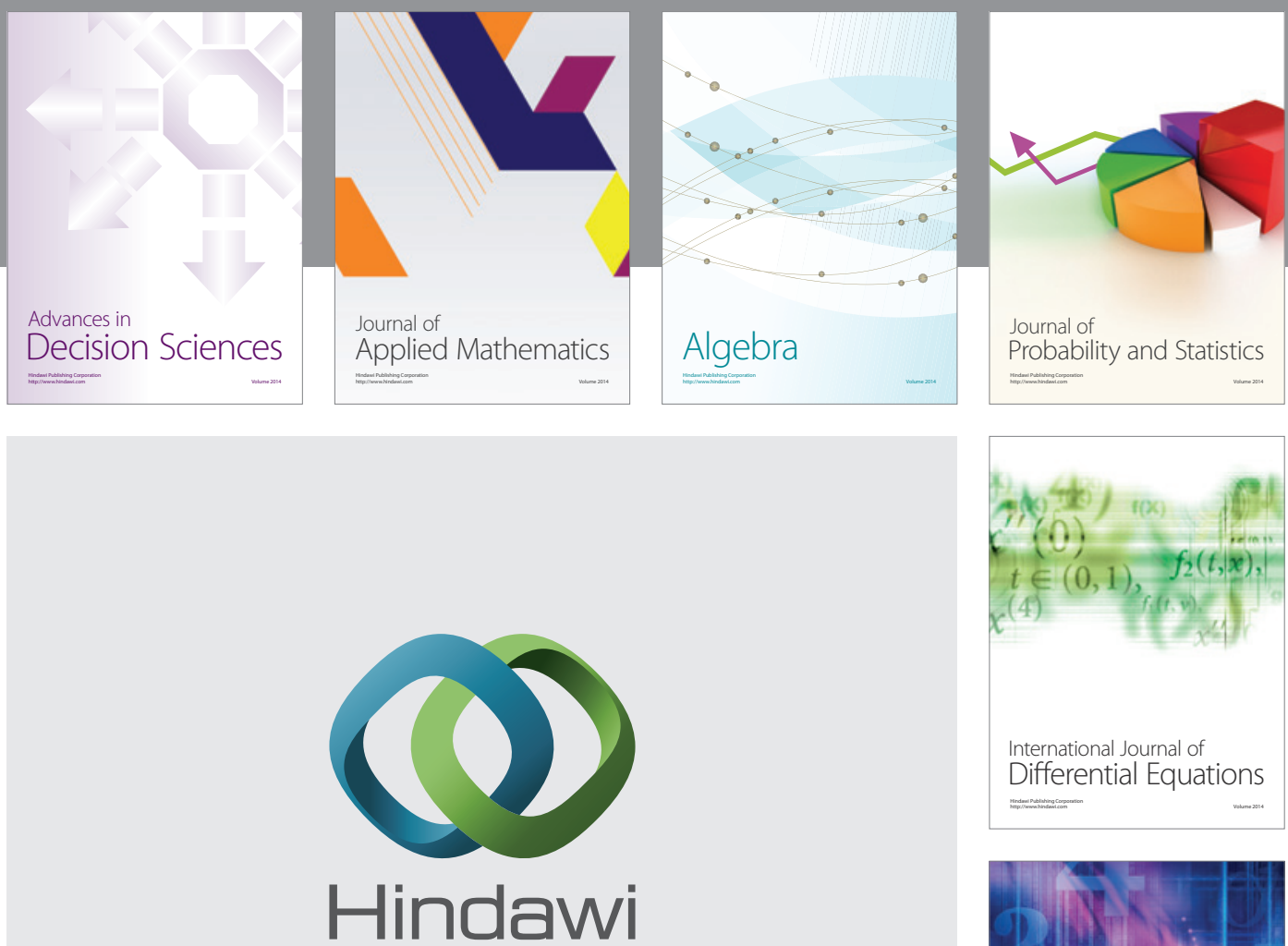

Submit your manuscripts at http://www.hindawi.com
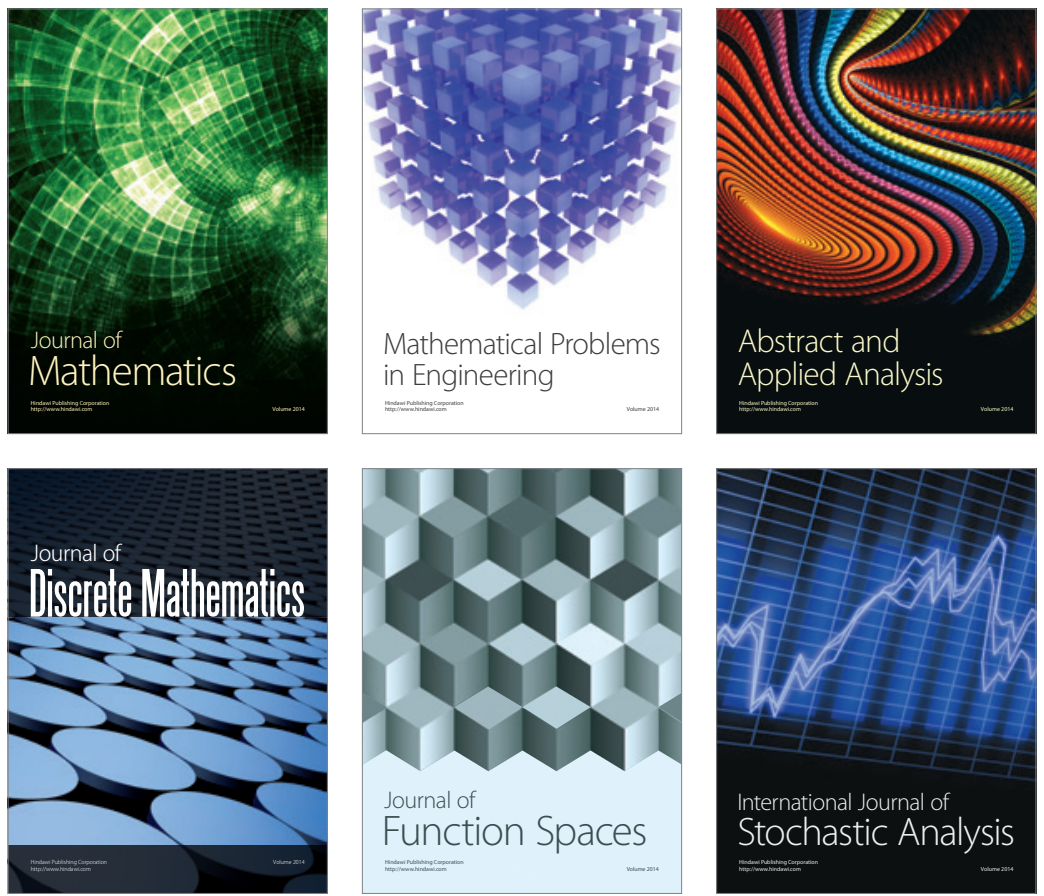

Journal of

Function Spaces

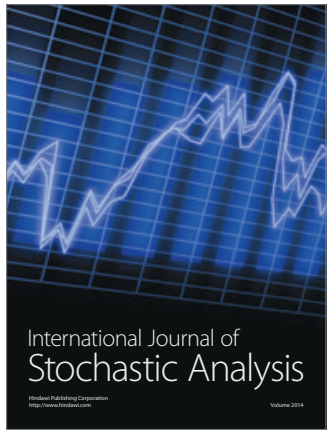

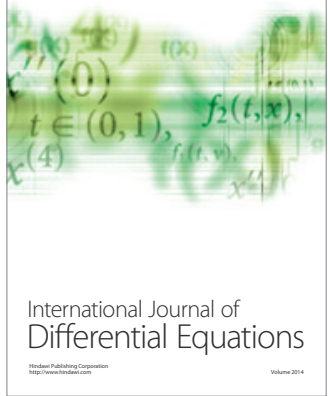
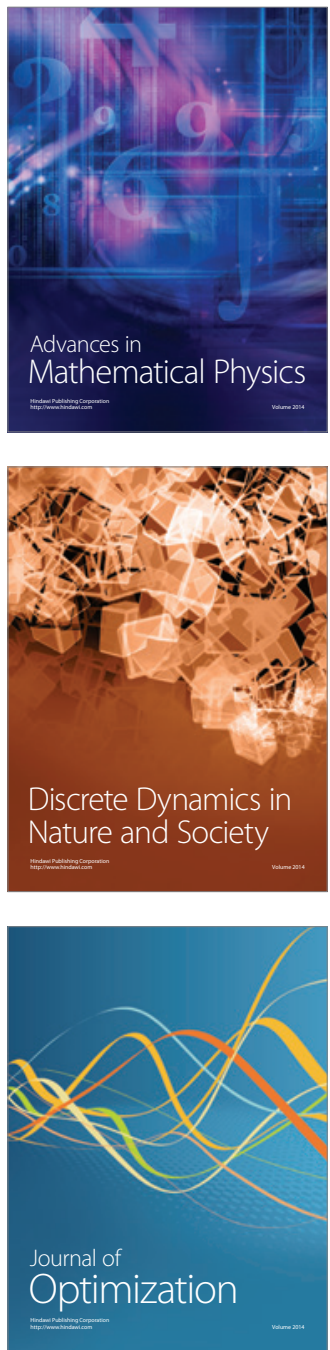